\title{
DECISION-MAKING AT DIFFERENT LEVELS OF RATIONALITY: SUBJECTS'S COGNITIVE, NEURAL AND PSYCHO-DYNAMIC CHARACTERISTICS
}

\footnotetext{
Dr. Belykh Tatiana Viktorovna, Department of Psychology, Saratov National Research University named after N.G. Chernyshevsky, Russian Federation Email: tvbelih@mail.ru

Dr. Grishina Nina Pavlovna, Department of Psychology, Saratov National Research University named after N.G. Chernyshevsky, Russian Federation

Email: grishinaninapavlovna@gmail.com

Dr. Zinchenko Ekaterina Mokhailovna, Department of Psychology, Saratov National Research University named after N.G. Chernyshevsky, Russian Federation

Email: odonata1108@yandex.ru

ARTICLE INFO

Original Research

Received: September, 15.2017.

Revised: December, 05.2017.

Accepted: January, 11.2018.

doi:10.5937/ijcrsee1801039B

UDK

005.5:159.9

\begin{abstract}
A B S T R A C T
In modern science, the issue of studying the decision-making process is challenging. If these decisions are connected with finances: monetary gains or losses - the problem becomes even more acute. Decision-making is almost always connected with risk and uncertainty. In order to investigate the problem of financial decision-making at risk laboratory studies have been conducted to determine the degree of rationality of such decisions as well as the neural and psychodynamic characteristics of the subject. This paper presents the results of the study of this problem at the extreme values of the probability of a gain or a loss -0.99 and 0.01 .
\end{abstract}

\author{
Keywords: \\ decision-making, \\ risk, \\ neural and psychodynamic \\ characteristics, \\ oculomotor activity.
}

(c) 2018 IJCRSEE. All rights reserved.

\section{INTRODUCTION}

The problem of research on decisionmaking process and its psychological components remains relevant to modern scientific knowledge. The first approaches to its study have been formulated in the works of J. Von Neumann and Morgenstern (1944) within the theory of games and economic behavior and the main stages of decision-making have been defined (building alternatives, evaluating them

Corresponding Author

Dr. Belykh Tatiana Viktorovna, Saratov National Research University named after N.G. Chernyshevsky, Russian Federation, Email: tvbelih@mail.ru

This work is licensed under a Creative Commons Attribution - NonCommercial - NoDerivs 4.0. The article is published with Open Access at www.ijcrsee.com and choosing the best alternative) (Morosanova and Indina, 2011). As a cross-cutting issue decision-making process has been explored both through a formalized research strategy, using mathematical and computer-based tools peculiar to economic and engineering sciences and using qualitative methods of analysis used in psychology.

The psychological researches of a decision-making problem conducted by Brushlinsky A. V. (1994), Velichkovskiy B. M. (2006), Gurova L. L. (1976), (1984), Zhuravlev A. A. (2005), Zhuravlev and Kupreychenko, (2003), Karpov A. V. (2000, January/February), Kitov A. I. (1983), Kornilova T. V. (2010), (2015), Lomov B. F. (1981), Lomov and Zhuravlev, (1978), Shadrikov V. D. (Shadrikov and Karpov, 1983), Kochetkov V. V. (2008), Skotinnikova I. G. (Kochetkov and Skotnikova, 1993) allowed to analyze a complex of not only situational decision-making determinants, but 
also individual and psychological ones. The works also focus on the identification of heuristic strategies for alternative choices. Decision-making is studied within psychology of management (V. A. Abchuk (2010), Kornilova T. V. (2010), V. V. Grigolova (1981), D. N. Zavalishina (Zavalishina, DN, Lomov, BF, and Rubakhin, VF., 1979), A. V. Karpov (2000, January/February), T. V. Kornilova (2005), V. V. Kochetkov (2008), I. Khol (1975), O. I. Larichev (Larichev, OI, Kortnev, AV, and Kochin, DY., 2002), B. F. Lomov (1981), E. V. Markova (Karpov and Markova, 2003), E. F. Rubahin (Zhuravlev, AP, Rubakhin, VF, and Shorin, VG., 1981), I. G. Skotnikova (Shendryapin and Skotnikova, 2015), L.N. Sumarokov (Sumarokov and Timofeeva, 1990), A. V. Fillipov (2001) and etc.) and new area of interdisciplinary knowledge - economic or financial behavior (Grishina, NP, Belykh, TV, and Sidorov, SP., 2015).

Cognitive psychology, by examining the decision-making process, aims to identify the characteristics of the strategy in experimental conditions and the way it is implemented, which are used by the subject on the basis of an analysis of information and orientation in situations. The choice of strategy, as well as the style of decision-making, may depend both on the structurally functional characteristics of personality and on the generated and potential cognitive factors (the nature of the information processing, the existence of cognitive capacity, cognitive and psychodynamic plasticity).

The purpose of the study is to identify individual differences in oculomotor activity, neural and psychodynamic characteristics of personality that determine the rational/irrational way of making a financial decision and the preference for subject of risk or guaranteed gain in the modelled conditions.

\section{MATERIALS AND METHODS}

In this study the formalized interviewing technique and the procedure of the laboratory experiment were applied to detect the intraindividual differences on the neurodynamic and psychodynamic level of the integral personality, depending on the respondents' preference for guaranteed success or risk when making financial decisions.

The methodology of E.P.Ilyin is a tapping test, which reveals the characteristics of the neural system properties with the help of psychomotor indicators (Eliseeva, 2003). The questionnaire "Formally-dynamic personal properties" V. M. Rusalova is aimed at identifying the psychodynamic features in the intellectual, communicative and psychomotor spheres. The laboratory experiment is used to study the financial decision-making process under modelled conditions.

In order to achieve the goal of the study a psychological and mathematical simulation was carried out of financial decision-making situations with different degrees of risk, using a hardware method to register the movement of an eye activity with the help of the eye tracking system, a model RED 500 System (SensoMotorik Instruments $\mathrm{GmbH}$ ). 76 people aged 18-50 years took part in the study. The group of experts whose results we compare with the general sample of the students was singled out among the subjects.

Kahneman and Tversky's prospect theory is used as the basis for our experiment (Kahneman and Tversky, 1979). The subjects were consistently faced with a choice between two alternatives, one of which was a probability assessment of the risk to lose or gain a certain amount of money and the other one was either sure gain or sure loss of more/less certain amount of money. As a risk alternative we took the prospects offered by Kahneman and Tversky (Tversky and Kahneman, 1992). A guaranteed option is a risk-free outcome in a particular monetary equivalent. The amount of monetary equivalent is fixed in a similar way as the study of Kahneman and Tversky as logarithmically distributed between the extreme values perspective outcomes. For example, "99\% chance to win nothing and 1\% chance to win \$200" have values of a logarithmic function distributed between 0 and $\$ 200$. The number of guaranteed values was reduced from the initial seven to five, as the goal of setting a threshold for risk-aversion or risk seeking as well as loss aversion is not fundamental for our study.

The logarithmic distribution takes into account probability assessment and offers values that are comparable to the risk option in terms of common sense.

The definition of the risk perspective itself was also modified. We switched from the term "odds" to the term "probability" and we used shares instead of percent.

Having two alternatives the subject has the information about preliminary calculated expected value and dispersion (as a risk measure) for the given choice, which allows to assess the degree of every subjects' rationality when making decisions. 
The article presents results for the extreme probability of a gain or a loss - 0.99 and 0.01 .

The results were carefully analyzed and distributed to the groups with different degrees of rationality. Our analysis uses the following definitions of specific decisions:

- rational $(+)$

- risk- averting (RA)

- risk-seeking (RS)

- loss-averting (LS)

- irrational (-)

In order to determine group membership the expected value and dispersion (risk measure) of the risk option are compared with the same parameters of the guaranteed outcome.

Table 1. Comparison of expected value and dispersion of the risk option with the guaranteed outcome in win-win situations

\begin{tabular}{lcccc}
\hline & + & $\mathrm{RA}$ & $\mathrm{RS}$ & - \\
\hline Expected & $>=$ & $<$ & $>$ & $<$ \\
value & & & & $=$ \\
\hline Dispersion & $<$ & $<$ & $>$ & $>$ \\
\hline
\end{tabular}

Table 2. Comparison of expected value and dispersion of the risk option with the guaranteed outcome in lose-lose situations

\begin{tabular}{lllll}
\hline & + & RA & LA & - \\
\hline Expected & $>$ & $<=$ & $>=$ & $<$
\end{tabular}

value

Dispersion $<<>>$

After detailed analysis of the decisions of each subject, it is necessary to distribute the entire sample across groups according to the rationality of the decisions. According to Markowitz (1952), a rational investor prefers more to less and certainty to uncertainty. Thus, we believe that the percentage of rationality is a combination of rational and risk-averting decisions in the total number of the offered choices ( + and RA).

Then all the subjects are divided into three groups:

- rational only ( $>60 \%$ of rationality)

- marginal (60-40\% of rationality)

- irrational only $(<40 \%$ of rationality)

The same procedure is used to determine the rationality of decisions in subgroups of financial decision-making:
- gains with a probability 0.99

- gains with a probability 0.01

- losses with a probability 0.99

- losses with a probability 0.01

\section{RESULTS}

The conducted study has revealed the intra-individual differences in financial decision-making with different degree of risk or preference of receiving the guaranteed success.

The respondents who prefer the guaranteed outcome have the most stable and medium strong type of nervous system that allows to sustain high levels of activity or to maintain the mobilization of efforts at the rate, which is optimal for human body

The respondents who are risk-takers mostly have the stable type of nervous system, but also there are represented weak, medium weak and medium strong types of nervous system. It reflects greater variability of typological nervous system properties of the respondents of this group. And also it suggests that risk acceptance may be a result of the weak capacity to maintain high level of suspension during making a choice.

When comparing the psychodynamic characteristic, the two samples revealed differences in indicators such as ergicity, plasticity and intelligent velocity. These indicators are more conspicuous in a group of respondents with a preference for risk when making a financial decision. At the same time intellectual emotionality characterized as lability in emotions' display is more common to the respondents who prefer the guaranteed outcome when making financial decisions.

In order to identify latent factors of cognitive processing in decision-making the peculiarities of the oculomotor activity were under investigation on the second stage of the study, furthermore the subjects were divided into three groups by degree of decision-making rationality, which has been identified by the ratio of preferred risk in each of the situations with the help of a mathematical expectation indicator. Three groups of respondents were identified during the experiment: 1 group -rational (25\%), 2 group - marginal (42\%), 3 group-irrational $(33 \%)$.

The percentage of rationality of the adopted financial decisions showed that the largest number of subjects represents the marginal group for the entire sample, for the sample excluding experts as well as in the expert group. 
(IJCRSEE) International Journal of Cognitive Research in Science, Engineering and Education Vol. 6, No.1, 2018.

Table 3. Results of testees' distribution for groups depending on the rationality of the adopted decisions

\begin{tabular}{lcccccc}
\hline & \multicolumn{2}{c}{ Entire sample } & \multicolumn{2}{c}{$\begin{array}{c}\text { Sample } \\
\text { excluding } \\
\text { experts }\end{array}$} & \multicolumn{2}{c}{ Experts } \\
\cline { 2 - 7 } & Number & $\%$ & Number & $\%$ & Number & $\%$ \\
\hline $\begin{array}{l}\text { Rational } \\
\text { only }\end{array}$ & 18 & 23 & 15 & 21 & 3 & 27 \\
\hline Marginal & 33 & 43 & 26 & 41 & 7 & 63 \\
\hline $\begin{array}{l}\text { Irrational } \\
\text { only }\end{array}$ & 25 & 32 & 24 & 36 & 1 & 9 \\
\hline Total & 76 & 100 & 65 & 100 & 11 & 100 \\
\hline
\end{tabular}

In the analysis of the results we also studied rationality in subgroups to obtain more information about the conditions in which the subjects are the most irrational. An analysis of the rationality of decisions in subgroups of financial decision-making situations has shown that the greatest cumulative percent of rationality corresponds to a win-win situation with the probability of 0.99 . Further there are subgroups of a lose-lose situation with probability 0.01 and a win-win situation with probability 0.99 . The least rationality is noted in the subgroup of loss situations with the probability of 0.99 .

It can be concluded that an extremely high probability of a loss has a strong impact on the rationality of financial decision-making. The data obtained in the subgroups allow to carry out a more profound study of the conditions of test subjects' sustainability.

The results of a statistical analysis according to Mann-Whitney criterion revealed differences in oculomotor activity among people with different degrees of rationality.

The data expressed in the oculomotor activity of the decision maker and their significant statistical differences in the compared groups are represented in Table 4.
Table 4. The oculomotor activity indicators of the respondents with different level of rationality (making financial decision with probability 0.01 and 0.99 )

\begin{tabular}{|c|c|c|c|c|c|c|}
\hline & Indicators & Rational & Irrational & & Marginal & \\
\hline 1 & End Time [ms] & 7609,317 & 9839,272 & $\circ$ & 9478,021 & $\circ$ \\
\hline 2 & Blink Count & 1,937 & 3,73 & $\circ$ & 4,23 & $\circ \Delta$ \\
\hline 3 & Blink Frequency [count/s] & 0,247 & 0,35 & $\circ$ & 0,41 & $\circ \Delta$ \\
\hline 4 & Blink Duration Total [ms] & 537,496 & 812,841 & $\circ$ & 998,493 & $\circ \Delta$ \\
\hline 5 & Blink Duration Average [ms] & 256,67 & 189,35 & $\circ$ & 187,72 & \\
\hline 6 & Blink Duration Minimum [ms] & 155,94 & 97,95 & $\circ$ & 87,96 & $\circ \Delta$ \\
\hline 7 & Fixation Count & 23,751 & 29,49 & $\circ$ & 28,71 & $\circ$ \\
\hline 8 & Fixation Frequency [count/5] & 3,097 & 3,031 & $\circ$ & 3,037 & $\circ \Delta$ \\
\hline 9 & Fixation Duration Total [ms] & 4253,019 & 5026,018 & $\circ$ & 5109,882 & $O \Delta$ \\
\hline 10 & Fisation Duration Average [ms] & 168,515 & 171,904 & & 183,396 & $\Delta$ \\
\hline 11 & Firation Duration Mas,imum [ms] & 510,54 & 409,55 & $\circ$ & 508,64 & $\Delta$ \\
\hline 12 & Fişation Duration Minimum [ms] & 58,99 & 57,25 & $\circ$ & 59,63 & $\Delta$ \\
\hline 13 & Fișation Dispersion Total [ps] & 1616,535 & 2304,447 & $\circ$ & 2103,124 & $\circ$ \\
\hline 14 & Fixation Dispersion Average $[p x]$ & 65,27 & 79,02 & $\circ$ & 110,83 & $\Delta$ \\
\hline 15 & Fisation Dispersion Maximum [ps] & 273,83 & 372,4 & 0 & 451,42 & \\
\hline 16 & Fixation Dispersion Minimum [p. $]$ & 36,74 & 26,97 & & 21,21 & $\circ \Delta$ \\
\hline 17 & Scanpath Length [px] & 5297,437 & 6935,024 & & 6530,591 & 0 \\
\hline 18 & Saccade Count & 42,217 & 59,75 & 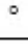 & 50,84 & $\Delta \Delta$ \\
\hline 19 & Saccade Frequency [count/9] & 5,549 & 5,828 & $\circ$ & 5.289 & $\Delta \Delta$ \\
\hline 20 & Saccade Duration Total [ms] & 2004,597 & 2696,002 & 0 & 2309,903 & $\Delta \Delta$ \\
\hline 21 & Saccade Duration Maximum [ms] & 112,94 & 114,78 & & 113,73 & $\Delta$ \\
\hline 22 & Saccade Duration Minimum [ms] & 23,2 & 22,79 & & 23,16 & $\Delta$ \\
\hline 23 & Saccade Amplitude Total ["] & 195,351 & 269,415 & & 251,71 & $\circ$ \\
\hline 24 & Saccade Amplitude Average [ $\left.{ }^{\circ}\right]$ & 5,14 & 5,21 & & 5,09 & 5 \\
\hline 25 & Saccade Amplitude Maximum ["] & 30,66 & 34,4 & & 35,87 & \\
\hline 26 & Saccade Velocity Total $[\mathrm{p} / \mathrm{s}]$ & 3365,475 & 4939,815 & & 4644,332 & 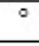 \\
\hline 27 & Saccade Velocity Maximum $\left[{ }^{\circ} / 5\right]$ & 276,19 & 379,42 & & 373,75 & \\
\hline 28 & Saccade Velocity Minimum [0/s] & 28,93 & 28,22 & & 33,65 & 4 \\
\hline 29 & Saccade Latency Average [ms] & 149,71 & 138,859 & & 155,931 & $\circ \Delta$ \\
\hline
\end{tabular}

Note: significant differences $\bigcirc$ - in comparison with the group 'rational'; $\Delta$ - in comparison with the group 'marginal', $p \leq 0,05$.

On the basis of the data provided, the respondents of the "rational" group in comparison with the marginal group spent less time to make a decision. The indicator - Scanpath Length, which is less in the group of rational respondents, also confirms this.

The maximum number, frequency and total duration of the blinks (Blink Frequency and Total Duration indicators) were most expressed by respondents demonstrating an irrational strategy for decision-making. This data implicitly indicates the increased emotion of the group's respondents, which determines the time taken to make decisions and reflects the specific style of its characteristics.

Both significant differences and similarities in fixation indicators were identified. The rational and irrational respondents have sig-nificantly more fixations rather than the marginal group. It indicates the similarities 
in the way the incoming information is processed - a thought delay in assessing choice alternatives. However, the "rational" respondents have considerably less number of fixations and their total and average dispersion. For members of the "irrational" group, the value of the overall and average duration of the fixation is clearly greater. This is different than in the first group, the information-processing strategy and the greater inclination to "Immersion" in the choice situation, which is related to the greater emotion.

Significant differences were found in three samples for saccades indicators. Thus, the number, total and maximum duration of the saccades, as well as the latent saccades period, are significantly higher at the "margin-al" respondents. They also have the smallest Saccade Duration Minimum indicator. The marginal respondents have the maximum frequency of saccades and the irrational re-spondents have the minimum frequency. The overall amplitude and the overall velocity of the saccades are faithfully lower among the representatives of the "rational" group, which shows less exposure to situational and emotional factors. This data confirms once again that there is a way for each group of comparisons to process information when making decisions that determine the differences in the time spent on decision-making and style preferences.

\section{DISCUSSIONS}

Cognitive psychology has long been studying the decision-making process. Today, the task of identification of the features of the strategy and the way it is implemented in reality is set in the experimental conditions. The subject of decision-making uses these strategies on the basis of analysis of the information presented and orientation in the situation. On the one hand, the choice of strategy, as well as the style of decision-making, can depend on the structural and functional characteristics of individuality. On the other hand, it depends on the formed and potential cognitive factors (the nature of information processing, the presence of cognitive potential, cognitive and psychodynamic plasticity).

The ability to identify such individual strategies or styles allows us in prospective to predict the degree of rationality of a solution in a risk environment. Also, we assume that these strategies can change with the course of life and the acquisition of different experiences. Being well aware of the mechanism of forming the style of decision-making, we can change it in order to improve the degree of rationality of future financial decisions.

\section{CONCLUSIONS}

Summarizing, it is important to note, that the study has revealed the important trends in the identification of the cognitive and intra-individual differences in financial decision-making with different degrees of risk or the preference for the guaranteed success.

It is revealed that emotional lability at intellectual data processing and also unequally probable combination of stable, weak, me-dium weak and medium strong types of nervous system are common to the respondents who prefer the guaranteed result when making financial decisions. Such a combination of splitlevel personality properties explains testees' preference for the guaranteed result in terms of greater expressed emotionality at the level of temperament and less energy endurance at the level of nervous system properties.

Intellectual endurance, high speed of intellectual information processing, intellectual plasticity in combination with stable and medium strong type of nervous system create conditions for taking risks in the context of economic behaviour.

The nature of the cognitive information processing at an assessment of alternatives in the modelled financial decision-making environment varies among respondents, depending on the expressed rationality/irrationality. The oculomotor activity implicitly reflects the strategy and style features of decision-making, which is reflected in the amount of time taken to make decisions, the degree of emotion when choosing the proposed alternatives, the degree of "immersion" into the situation. The identified trends suggest that a similar research model could be used to identify the intraindividual determinants of decision-making in the context of personal economic behavior.

\section{ACKNOWLEDGEMENTS}

We thank students of Saratov National Research University named after N.G. Chernyshevsky for participation in our empirical experiments. We also would like to thank our colleagues from Economical Department for great support and for comments that greatly improved the manuscript. 
(IJCRSEE) International Journal of Cognitive Research in Science, Engineering and Education

Vol. 6, No.1, 2018.

\section{Conflict of interests}

est.

The authors declare no conflict of inter-

\section{REFERENCES}

Abchuk, V. A. (2010). Organization management system. Schoolbook. Souz, St. Petersburg.

Brushlinsky, A. V. (1994). Problems of subject's psychology. IP RAS, Moscow.

Eliseeva, O. P. (2003). Determination of the coefficient of functional asymmetry and properties of the nervous system according to psychomotor indicators. Workshop on the psychology of personality. SPb, St. Petersburg.

Filippov, A. V. (2001). Signs of management activity. In Organizational psychology (Ed. Vinokurov, L. V, \& Skrypyuk, I. I). Peter, St. Petersburg, pp. 436-489.

Golubinov, V. V. (1991). Personality control of the criterion of psychophysical problem solution's optimality. In Problems of differential psychophysics. Publishing house of the IP RAS, Moscow, pp. 177-196.

Grigolava, V. V. (1981). Decision making in human activity in the light of the theory of activity Uznadze D. N. In Normative and descriptive models of decision-making (Ed. Lomov, B .F.). Nauka, Moscow, pp. 46-51.

Grishina, N. P, Belykh, T. V, \& Sidorov, S. P. (2015). The conceptual analysis as a methodological basis for the creation of a behavioural theory of the financial decision-making under uncertainty. International Annual Edition of Applied Psychology: Theory, Research, and Practice, 2(1), 5-10. https://cyberleninka.ru/article/n/the-conceptualanalysis-as-a-methodological-basis-for-the-creation-of-a-behavioural-theory-of-the-financialdecision-making-under

Gurova, L. L. (1976). Psychological analysis of problem solving. Publishing house of Voronezh University, Voronezh.

Gurova, L. L. (1984). Decision-making as a problem of psychology of cognition. Questions of psychology, 1, 125-131.

Kahneman, D., \& Tversky, A. (1979). Prospect theory: an analysis of decision under risk. Econometrica, 47, 263-291, http://www.jstor.org/stable/1914185

Karpov, A. V. (2000, January/February). Decisionmaking processes in the structure of managerial activity. Psychological journal, Ed. Brushlinsky, AV, Aleksandrov, IO, \& Chesnokova, II, 21 (1), 63-78.

Karpov, A. V, \& Markova, EV. (2003). Psychology of styles of management decisions. Open Society Institute, Yaroslavl.

Khol, I. (1975). Efficiency of managerial decisions. Progress, Moscow.

Kitov, A. I. (1983). Psychological features of managerial decisions-making. Znanie, Moscow.

Kochetkov, V. V, \& Skotnikova, I. G. (1993). Individual psychological problems of decision-making. Moscow.

Kochetkov, V. V. (2008). Conceptual approaches and theoretical models of foreign policy decisionmaking processes: critical analysis. Bulletin of Moscow University. Series 18. Sociology and
Political Science, 3, $100-113$

Kornilova, T.V. (2005). Methodological problems of decision-making psychology. Psychological Journal, 26 (1), 7-17.

Kornilova, T. V. (2010). The uncertainty principle in psychology: foundations and challenges. Psychological research: electron. sci. journal, 3 (11), http://psystudy.ru/num/2010n3-11/320kornilova11.html

Kornilova, T. V. (2015). Risk and decision-making: the psychology of uncertainty, http://www.rfh.ru/ downloads/Books/144693004.pdf

Larichev, O. I, Kortnev, A. V, \& Kochin, D. Y. (2002). Decision support system for classification of a finite set of multicriteria alternatives. Decision support systems, 33 (1), 13-21. https://doi. org/10.1016/S0167-9236(01)00132-4

Lomov, B. F, \& Zhuravlev, A. L. (1978). Psychology of management. Znanie, Moscow.

Lomov, B. F. (1981). Mathematics and psychology in the study of decision-making processes. Normative and descriptive models of decision-making. Moscow.

Markowitz, H. (1952). Portfolio selection. Journal of Finance, 7, 7-91, http://www.jstor.org/stable/2975974

Morosanova, V. I, \& Indina, T. A. (2011). Regulatory and personal basis for decision-making: Monograph. Nestor-Istoriya, St. Petersburg, Moscow.

Neumann, J., \& Morgenstern, O. (1944). Theory of games and economic behavior. University Press, Princeton, NJ.

Shadrikov, V. D, \& Karpov A. V. (1983). Psychological characteristics of the decision-making processes in managerial activity. Paper presented at the Mater. Vses. Conf. on the psychology of management.

Shendyapin, V. M, \& Skotnikova, I. G. (2015). Modeling of decision-making and confidence in sensory tasks. Institute of psychology of the Russian academy of sciences, Moscow.

Sumarokov, L. N, \& Timofeeva, O. V. (1990). If there is no computer ... Methods of organizing the work of the head. Publishing Standards, Moscow.

Tversky, A., \& Kahneman, D. (1992). Advances in prospect theory: cumulative representation of uncertainty. Journal of Risk and Uncertainty, 5, 297-323, http://scholar.google.com/ scholar?as $\mathrm{q}=\mathrm{A}+$ Question $+\mathrm{of}+$ Control: + Alte rnative+Patterns + of + Air + Conditioner+Use \& as authors $=$ Lutzenhiser

Velichkovsky, B. M. (2006). Cognitive science. Fundamentals of the psychology of cognition. (In 2 volumes). Information Center "Akademiya", Moscow.

Zavalishina, D. N, Lomov, B. F, \& Rubakhin, V. F. (1979). Levels and stages of decision-making. In Problems of Decision Makin. Nauka, Moscow, pp. 16-32.

Zhuravlev, A. P, Rubakhin, V. F, \& Shorin, V. G. (1981). Management work and efficiency of social production. Science and Technology, Minsk.

Zhuravlev, A. L. \& Kupreychenko A. B. (2003). Moral and psychological regulation of economic activity. Publishing house "Institute of Psychology of RAS", Moscow.

Zhuravlev, A. L. (2005). Psychology of mutual activ-ity. Publishing house "Institute of Psychology of the Russian Academy of Sciences", Moscow. 